\title{
Can it be better? \\ EMLA before peripheral venous cannulation of children
}

\author{
Stojanović Stipić S. ${ }^{1}$, E. Tatkovic ${ }^{2}$, Z. Nincevic ${ }^{1}$, M. Gabelica ${ }^{3}$, J. Nincevic ${ }^{4}$, R. Gabelica ${ }^{5}$
}

${ }^{1}$ Department of Anaesthesiology \& Intensive Care, Split, (Croatia), ${ }^{2}$ Community Health Centre dr.Ante Franulović - Korcula (Croatia), ${ }^{3}$ Department of Neurology,Split (Croatia), ${ }^{4}$ Institute of Public Health SDŽ - Split (Croatia), ${ }^{5}$ Department of Anaesthesiology and Intensive Care Medicine, Zagreb (Croatia),

\section{Background and Goal of Study:}

The introduction of i.v. cannula is one of most stressful situations during hospitalization of children. Generally, local anaesthetics EMLA is used to minimize this fear. The aim of this study was to evaluate the reaction of introduction of venous access with FLACC scale and to determine the optimum time of application of local anaesthetic EMLA prior to intravenous cannula insertion.

\section{Materials and Methods:}

This study was approved by the institute ethics committee of our hospital and informed consent was obtained from parents before surgery. 120 children were included in total (aged 0-10, ASA I-II) and they were divided into two equally large groups: younger ( 0 to 4.99 ) and older (5 to 9.99) years. Each group had three subgroups of subjects who had the EMLA applied on the site of planned i.v. cannula insertion. In the first group, EMLA was applied 15 minutes before setting up i.v. cannula, and in the second and third group, 30 and 45 minutes before, respectively. Children who had a history of allergy to local anaesthetics or any components of the EMLA cream, uncertain drug sensitivities, open wound at the application site or severe systemic disease, were excluded. The cream was applied over a prominent vein on the dorsum of the hand in a thick layer and covered with occlusive dressing.

\section{Results and Discussion:}

No significant differences were found in either age group regarding pain relief in terms of duration of EMLA before the introduction of i.v. cannula.

The heart rate was statistically significantly higher in girls compared to boys before and during i.v. cannulation ( $\mathrm{t}=$ $2.541 ; \mathrm{df}=118 ; \mathrm{p}=0.012$ ). There was also a statistically significant difference in age $(\mathrm{t}=5.292 ; \mathrm{df}=118 ; \mathrm{p}$ $<0.001)$. Heart beats were higher in the younger age group. The oxygen saturation was also statistically significantly lower with girls before $(t=2,541$; $d f=118 ; p$ $=0,012)$ and during ( $t=5,292 ; d f=118 ; p<0,001)$ induction of iv route.

Researching specific parameters of the FLACC scale according to gender, there were no statistically significant differences. However, it can be noted that boys have a worse reaction to pain.

\section{Conclusion:}

We should avoid longer application of EMLA because longer exposure to EMLA has no statistically significant effect on pain relief. Shorter application reduces the risk of possible side effects and reduces the time and preparation for surgery.

\begin{tabular}{|c|c|c|c|c|c|c|c|c|c|c|c|c|}
\hline \multirow{3}{*}{ 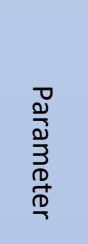 } & \multirow[b]{3}{*}{ FLACC } & \multicolumn{9}{|c|}{ EMLA application length } & \multirow{2}{*}{\multicolumn{2}{|c|}{$\begin{array}{l}\text { Total } \\
f(\%)\end{array}$}} \\
\hline & & \multicolumn{3}{|c|}{$\begin{array}{l}15 \text { minutes } \\
f(\%)\end{array}$} & \multicolumn{3}{|c|}{$\begin{array}{c}30 \text { minutes } \\
\mathrm{f}(\%)\end{array}$} & \multicolumn{3}{|c|}{$\begin{array}{c}45 \text { minutes } \\
f(\%)\end{array}$} & & \\
\hline & & $\begin{array}{c}\text { Younger } \\
<5 \text { yrs }\end{array}$ & $\begin{array}{l}\text { Older } \\
>5 y r s\end{array}$ & Total & $\begin{array}{c}\text { Younger } \\
<5 \text { yrs }\end{array}$ & $\begin{array}{l}\text { Older } \\
>5 \mathrm{yrs}\end{array}$ & Total & $\begin{array}{c}\text { Younger } \\
<5 y r s\end{array}$ & $\begin{array}{l}\text { Older } \\
>5 y \text { ys }\end{array}$ & Total & $\begin{array}{c}\text { Younger } \\
<5 \text { yrs }\end{array}$ & $\begin{array}{l}\text { Older } \\
>5 y \mathrm{ys}\end{array}$ \\
\hline \multirow{4}{*}{ 華 } & 0 & $5(25,0)$ & $13(65,0)$ & $18(45,0)$ & $3(15,0)$ & $9(45,0)$ & $12(30,0)$ & $0(0,0)$ & $12(60,0)$ & $12(30,0)$ & $8(13,3)$ & $34(56,7)$ \\
\hline & 1 & $8(40,0)$ & $6(30,0)$ & $14(25,0)$ & $11(55,0)$ & $10(50,0)$ & $21(52,5)$ & $10(50,0)$ & $8(40,0)$ & $18(45,0)$ & $29(48,3)$ & $24(40,0)$ \\
\hline & 2 & $7(35,0)$ & $\begin{array}{c}1 \\
(5,0)\end{array}$ & $\begin{array}{c}8 \\
(20,0)\end{array}$ & $6(30,0)$ & $\begin{array}{c}1 \\
(5,0)\end{array}$ & $7(17,5)$ & $10(50,0)$ & $\begin{array}{c}0 \\
(0,0)\end{array}$ & $10(25,0)$ & $23(38,3)$ & $\stackrel{2}{2}$ \\
\hline & Total & $20(100,0)$ & $20(100,0)$ & $40(100,0)$ & $20(100,0)$ & $20(100,0)$ & $40(100,0)$ & $20(100,0)$ & $20(100,0)$ & $40(100,0)$ & $60(100,0)$ & $\begin{array}{c}60 \\
(100,0) \\
\end{array}$ \\
\hline \multirow{4}{*}{$\begin{array}{c}\text { Do } \\
\text { og } \\
\text { w }\end{array}$} & 0 & $5(25,0)$ & $16(80,0)$ & $21(52,5)$ & $7(35,0)$ & $14(70,0)$ & $21(52,5)$ & $9(45,0)$ & $13(65,0)$ & $22(55,0)$ & $21(35,0)$ & $43(71,7)$ \\
\hline & 1 & $8(40,0)$ & $2(10,0)$ & $10(25,0)$ & $10(50,0)$ & $\begin{array}{c}6 \\
(30,0)\end{array}$ & $16(40,0)$ & $8(40,0)$ & $7(35,0)$ & $15(37,5)$ & $26(43,3)$ & $15(25,0)$ \\
\hline & 2 & $7(35,0)$ & $2(10,0)$ & $9(22,5)$ & $3(15,0)$ & $0(0,0)$ & $3(7,5)$ & $3(15,0)$ & $0(0,0)$ & $3(7,5)$ & $13(21,7)$ & $\begin{array}{c}2 \\
(3,3)\end{array}$ \\
\hline & Total & $20(100,0)$ & $20(100,0)$ & $40(100,0)$ & $20(100,0)$ & $20(100,0)$ & $40(100,0)$ & $20(100,0)$ & $20(100,0)$ & $40(100,0)$ & $60(100,0)$ & $\begin{array}{c}60 \\
(100,0)\end{array}$ \\
\hline \multirow{4}{*}{ 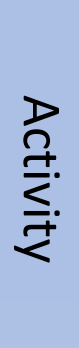 } & 0 & $\begin{array}{c}3 \\
(15,0)\end{array}$ & $15(75,0)$ & $18(45,0)$ & $\begin{array}{c}4 \\
(20,0)\end{array}$ & $14(70,0)$ & $18(45,0)$ & $\begin{array}{c}1 \\
(5,0)\end{array}$ & $14(70,0)$ & $15(37,5)$ & $\begin{array}{c}8 \\
(13,3)\end{array}$ & $43(71,7)$ \\
\hline & 1 & $\begin{array}{c}9 \\
(45,0)\end{array}$ & $\begin{array}{c}2 \\
(10,0)\end{array}$ & $11(27,5)$ & $10(50,0)$ & $\begin{array}{c}5 \\
(25,0)\end{array}$ & $15(37,5)$ & $15(75,0)$ & $\begin{array}{c}6 \\
(30,0)\end{array}$ & $21(52,5)$ & $34(56,7)$ & $13(21,7)$ \\
\hline & 2 & $\begin{array}{c}8 \\
(40,0)\end{array}$ & $\begin{array}{c}3 \\
(15,0)\end{array}$ & $11(27,5)$ & $\begin{array}{c}6 \\
(30,0)\end{array}$ & $\begin{array}{c}1 \\
(5,0)\end{array}$ & $\begin{array}{c}7 \\
(17,5)\end{array}$ & $\begin{array}{c}4 \\
(20,0)\end{array}$ & $\begin{array}{c}0 \\
(0,0)\end{array}$ & $\begin{array}{c}4 \\
(10,0)\end{array}$ & $18(30,0)$ & $\begin{array}{c}4 \\
(6,7)\end{array}$ \\
\hline & Total & $20(100,0)$ & $20(100,0)$ & $40(100,0)$ & $20(100,0)$ & $20(100,0)$ & $40(100,0)$ & $20(100,0)$ & $20(100,0)$ & $40(100,0)$ & $60(100,0)$ & $60(100,0)$ \\
\hline \multirow{4}{*}{ 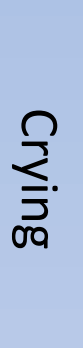 } & 0 & $\begin{array}{c}6 \\
(30,0)\end{array}$ & $16(80,0)$ & $22(55,0)$ & $\begin{array}{c}5 \\
(25,0)\end{array}$ & $13(65,0)$ & $18(45,0)$ & $\begin{array}{c}2 \\
(10,0)\end{array}$ & $17(85,0)$ & $19(47,5)$ & $13(21,7)$ & $46(76,7)$ \\
\hline & 1 & $\begin{array}{c}5 \\
(25,0)\end{array}$ & $\begin{array}{c}2 \\
(10,0)\end{array}$ & $\begin{array}{c}7 \\
(17,5)\end{array}$ & $\begin{array}{c}6 \\
(30,0)\end{array}$ & $\begin{array}{c}6 \\
(30,0)\end{array}$ & $12(30,0)$ & $10(50,0)$ & $\begin{array}{c}3 \\
(15,0)\end{array}$ & $13(32,5)$ & $21(35,0)$ & $11(18,3)$ \\
\hline & 2 & $\begin{array}{c}9 \\
(45,0)\end{array}$ & $\begin{array}{c}2 \\
(10,0)\end{array}$ & $11(27,5)$ & $\begin{array}{c}9 \\
(45,0)\end{array}$ & $\begin{array}{c}1 \\
(5,0)\end{array}$ & $10(25,0)$ & $\begin{array}{c}8 \\
(40,0)\end{array}$ & $\begin{array}{c}0 \\
(0,0)\end{array}$ & $\begin{array}{c}8 \\
(20,0)\end{array}$ & $26(43,3)$ & $\begin{array}{c}3 \\
(5,0)\end{array}$ \\
\hline & Total & $20(100,0)$ & $20(100,0)$ & $40(100,0)$ & $20(100,0)$ & $20(100,0)$ & $40(100,0)$ & $20(100,0)$ & $20(100,0)$ & $40(100,0)$ & $60(100,0)$ & $60(100,0)$ \\
\hline \multirow{4}{*}{ 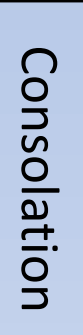 } & 0 & $\begin{array}{c}5 \\
(25,0)\end{array}$ & $15(75,0)$ & $20(50,0)$ & $\begin{array}{c}7 \\
(35,0)\end{array}$ & $18(90,0)$ & $25(62,5)$ & $8(40,0)$ & $18(90,0)$ & $26(65,0)$ & $20(33,3)$ & $51(85,0)$ \\
\hline & 1 & $\begin{array}{c}9 \\
(45,0)\end{array}$ & $\begin{array}{c}4 \\
(20,0)\end{array}$ & $13(32,5)$ & $\begin{array}{c}9 \\
(45,0)\end{array}$ & $\begin{array}{c}2 \\
(10,0)\end{array}$ & $11(27,5)$ & $10(50,0)$ & $\begin{array}{c}2 \\
(10,0)\end{array}$ & $12(30,0)$ & $28(46,7)$ & $\begin{array}{c}8 \\
(13,3)\end{array}$ \\
\hline & 2 & $\begin{array}{c}6 \\
(30,0)\end{array}$ & $\begin{array}{c}1 \\
(5,0)\end{array}$ & $\begin{array}{c}7 \\
(17,5)\end{array}$ & $\begin{array}{c}4 \\
(20,0)\end{array}$ & $\begin{array}{c}0 \\
(0,0)\end{array}$ & $\begin{array}{c}4 \\
(10,0)\end{array}$ & $\begin{array}{c}2 \\
(10,0)\end{array}$ & $\begin{array}{c}0 \\
(0,0)\end{array}$ & $\begin{array}{c}2 \\
(5,0)\end{array}$ & $12(20,0)$ & $\begin{array}{c}1 \\
(1,7)\end{array}$ \\
\hline & Total & $20(100,0)$ & $20(100,0)$ & $40(100,0)$ & $20(100,0)$ & $20(100,0)$ & $40(100,0)$ & $20(100,0)$ & $20(100,0)$ & $40(100,0)$ & $60(100,0)$ & $60(100,0)$ \\
\hline
\end{tabular}

Download PDF and read later

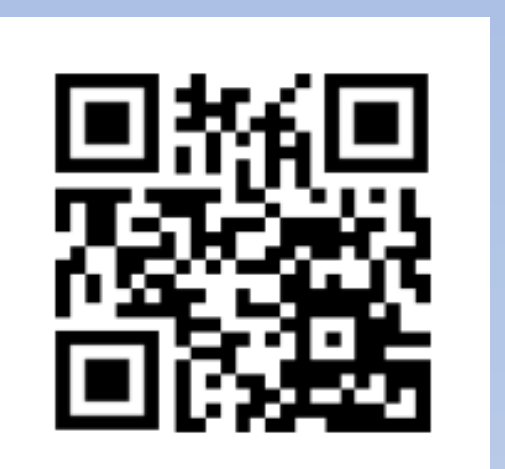

Copyright (C) 2018 Sanda Stojanovic Stipic, MD, PhD sandastojanovicstipic@gmail.com 